\title{
体内多肽自组装纳米材料在非肿瘤 相关疾病诊疗中的研究进展
}

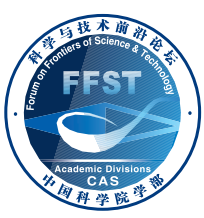

曾祥仲 ${ }^{1,2}$, 安红维 ${ }^{2,3}$, 王浩 ${ }^{2 *}$

1. 北京大学前沿交叉学科研究院, 北京 100871

2. 国家纳米科学中心, 中国科学院纳米生物效应与安全性重点实验室, 中国科学院纳米科学卓越创新中心, 北京 100190

3. 中国科学院高能物理研究所, 北京 100049

*通讯作者, E-mail: wanghao@nanoctr.cn

收稿日期：2019-02-20; 接受日期：2019-04-17; 网络版发表日期：2019-05-17

国家自然科学基金(编号: 51573032)和中国博士后科学基金(编号: 2018M631574)资助项目

摘要基于“活体自组装”策略发展的体内多肽自组装纳米探针和纳米药物, 具有高的特异性和生物利用度, 被 广泛应用于肿瘤的诊断和治疗方面. 基于体内多肽自组装纳米材料的独特优势, 目前也已经被用于肿瘤诊断与治 疗之外的其他生物医学领域, 如对细菌感染的精准检测、组织修复和神经性退行疾病的治疗. 本文主要对体内多 肽自组装纳米材料在非肿瘤诊疗之外的其他重大疾病诊疗中的应用进行了综述.

关键词活体自组装, 纳米材料, 多肽, 非肿瘤相关疾病

\section{1 引言}

多肽是介于氨基酸和蛋白之间的一类生物活性物 质，由多种氨基酸按照一定的顺序通过酰胺键连接而 成. 通常情况下，多肽是长度小于或等于 50 个氨基酸 的短链. 自然界中存在的氨基酸有 20 多种，每个氨基 酸都有类似的骨架结构和全然不同的R侧基．通过合 理的设计和多样化的合成手段可以产生多种功能性多 肽 ${ }^{[1 \sim 3]}$ ，这些功能性多肽虽然体积小、结构简单，但是 通常具有与天然蛋白类似的生物学功能 ${ }^{[3]}$. 相对于传 统的生物医用材料，多肽具有良好的生物相容性和潜 在的生物降解性 ${ }^{[4]}$. 多肽在自组装纳米生物材料方面 显示出巨大的潜力, 应用在组织工程、药物缓释等方
面 ${ }^{[5 \sim 9]}$. 正是由于以上优点, 多肽类生物材料已经成为 材料学和医学领域研究的热点 ${ }^{[5-9]}$.

目前, 研究人员主要是通过氢键、范德华力和静 电等弱相互作用力在溶液中构筑多肽自组装的纳米生 物材料, 进而在细胞和动物水平进行生物效应的评 价 ${ }^{[10,11]}$. 然而, 当多肽自组装纳米材料进入复杂的生 理环境后，纳米材料结构的稳定性、蛋白冠的形成等 诸多问题给相应的生物研究带来了更多的困难和挑 战 ${ }^{[12,13]}$. 近年来, 本课题组 ${ }^{[14]}$ 在多肽自组装的基础上, 提出了“活体自组装”策略使多肽自组装纳米材料在纳 米生物医学领域的应用进入了一个新的阶段. “活体自 组装”策略是指外源性的小分子在特定的病生理部位, 通过刺激响应后原位自组装形成高级组装体，进而发

引用格式: Zeng X Z, An H W, Wang H. Progress of in vivo self-assembled peptide nanomaterials for theranostics of non-cancerous diseases. Sci Sin Chim, 2019, 49: 1213-1219, doi: 10.1360/SSC-2019-0021 
挥生物功能. 体内多肽自组装纳米材料在肿瘤的诊断 和治疗等领域取得了众多研究成果 ${ }^{[15 \sim 17]}$. 目前已有很 多相关综述对此领域进行了报道 ${ }^{[15 ~ 17]}$. 但是，关于体 内多肽自组装纳米材料在细菌感染的检测、神经性退 行疾病的治疗和组织修复等非肿瘤相关疾病应用的综 述却鲜有报道. 本文则希望通过综述传递近来研究活 体多肽自组装过程中的一些富有新意并具有借鉴意义 的研究成果.

\section{2 体内多肽自组装体用于细菌感染和离子 的检测}

在疾病检测中，利用模块化的多肽分子在病灶部 位原位自组装形成纳米组装体是一种很有实用性的研 究策略，通过对多肽的结构调控可以将靶向、刺激响 应、组装和信号分子等功能集中于一体. 首先，通过 改变靶向多肽序列可以实现对待测物质的特异性识 别 ${ }^{[18]}$; 而对响应部分的调控则能使目标多肽具有多种 刺激响应性，如酶响应、光响应、 $\mathrm{pH}$ 响应、活性氧响 应以及温度响应等 ${ }^{[16]}$ ，以提高该策略的特异性；偶联 的信号分子可以根据检测需要进行偶联，如荧光分 子、光声信号分子、核磁信号分子等 ${ }^{[8,13,14,18 \sim 20]}$. 模块 化的功能多肽分子在病灶部位通过响应刺激, 原位自 组装形成超分子自组装体，该策略既提高了检测的特 异性又提高了信号分子的生物利用度, 从而实现疾病 的精准检测.

多重耐药菌引发的感染是威胁人类健康的一个 严重的问题. 目前，新型抗生素的研发速度远远落后 于广泛耐药菌出现的速度, 在不久的将来我们可能面 临无药可用的境地，因此发展新型的细菌感染的早期 诊断工具尤为重要. 本课题组 ${ }^{[14,21,22]}$ 通过活体自组装 的策略实现了细菌的活体检测. 首先, 本课题组设计 了模块化的功能多肽分子: 万古霉素作为靶向单 元 $^{[21]}$, 可以特异性识别革兰氏阳性菌; PLGVRG作为 明胶酶的水解底物片段，能够被感染部位过表达的明 胶酶剪切; 焦脱镁叶绿酸- $\alpha$ 作为光声信号分子，可以 自组装形成 $J$ 型聚集体，通过聚集诱导滞留(assembly/ aggregation induced retention, AIR)效应提高了光声 信号分子的富集，实现了活体水平对革兰氏阳性菌的 检测. 此外，对于由金黄色葡萄球菌引起的慢性感染，
由于部分细菌能够潜伏在巨噬细胞内，抗生素对其束 手无策. 目前，针对该类型细菌感染的检测和治疗存 在着挑战 ${ }^{[14,21,22]}$. 通过相同的模块化分子设计, 实现了 对巨噬细胞中金黄色葡萄球菌的特异性检测. 功能分 子(MPC)引入甘露糖作为靶向单元特异性靶向巨噬细 胞, YVHDC作为半胱氨酸蛋白酶(Caspase-1)的水解 底物片段，在感染了金黄色葡萄球菌的巨噬细胞内， 被过度表达的Caspase-1剪切; 螯合 $\mathrm{Cu}^{2+}$ 紫红素 18 $\left(\mathrm{P}_{18}\right)$ 作为光声信号分子 ${ }^{[22]}$ (图1(a)). MPC 在进入体内 后，首先通过甘露糖靶向到巨噬细胞内，在感染了金 黄色葡萄球菌的巨噬细胞内, 分子被细胞内过度表达 的caspase-1酶进行响应剪切, 得到的残基自组装形成 纳米纤维(图1(b)). $\mathrm{P}_{18}$ 形成组装体后光谱发生红移, 组 装体在710 740 $\mathrm{nm}$ 处的光声信号强度显著高于未组 装体的信号强度, 利用 $710 \mathrm{~nm} / 680 \mathrm{~nm}$ 的信号比值可 以实现对感染部位巨埃细胞的金黄色葡萄球菌的检 测(图1(c e)). 该工作最大的亮点就是通过比率的手段 有效排除其他杂散信号的干扰，大大提高了信号检测 的准确性 ${ }^{[22]}$.

在研究多肽活体自组装的过程中，非天然氨基酸 的引入能够发展更多特殊生物功能的纳米生物材料. 在实际研究中，含有非天然氨基酸的多肽分子在活体 内原位自组装进行离子检测方面表现出了高特异性. 虽然以往的研究对于 $\mathrm{Hg}^{2+}$ 的毒性已经有了一定的认 识, 但是对于 $\mathrm{Hg}^{2+}$ 在生物体内的分布和循环过程仍需 要进一步研究, 目前还缺乏能在活体情况下对 $\mathrm{Hg}^{2+}$ 分 布进行有效检测的荧光探针 ${ }^{[23]}$. 针对此难题, Zhao课 题组 ${ }^{[24]}$ 利用配位作用诱导多肽自组装同时伴随着聚 集诱导发光效应(aggregation induced emission, AIE), 其对 $\mathrm{Hg}^{2+}$ 的检测具有很好的应用前景和借鉴意义. 他 们将一条含有 $\gamma$-谷氨酸的谷胱甘肽与具有AIE效应的 四苯基乙烯(tetraphenylethylene, PTE)进行偶联，利用 谷胱甘肽中半胱氨酸上的颈基和 $\gamma$-谷氨酸上羧基与 $\mathrm{Hg}^{2+}$ 的配位得到的络合物分子，该分子可以通过 $\pi-\pi$ 相 互作用堆叠形成超分子组装体，随着分子在原位富集 形成纳米纤维或扭曲形成更大的纤维(图2(a)). 利用 PTE具有的AIE效应，由苂光的强度变化实现对 HeLa 细胞和斑马鱼中 $\mathrm{Hg}^{2+}$ 分布的检测(图2(b, c)). 需要指出 的是, 由于人体细胞微环境内各种离子含量的复杂性, 如何提高该方法对目标离子的特异性是此类课题进行 深入开展所需要解决的问题. 
(a)

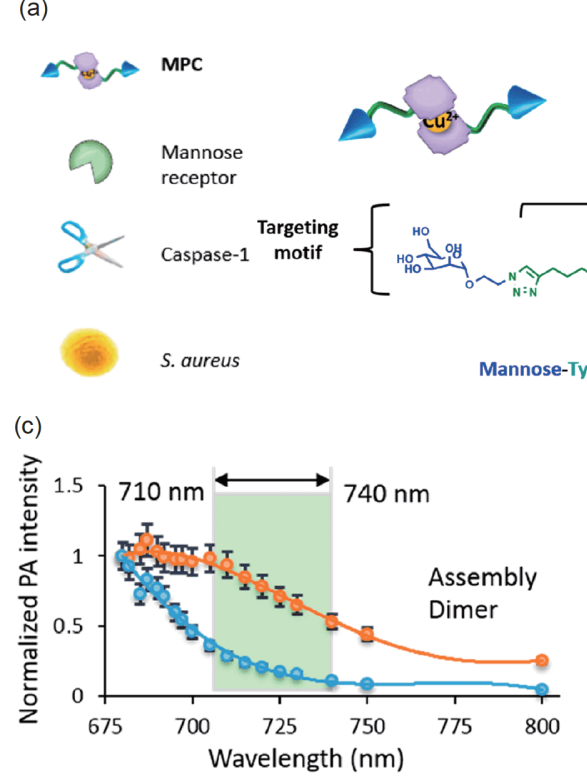

图 1 (a, b) MPC结构和MPC在感染部位作用示意图; MPC在体外(c)和体内 $(d, e)$ 实验表征 ${ }^{[22]}$ (网络版彩图)

Figure 1 (a, b) The chemical structure of MPC and the scheme of the interaction between MPC and infected tissue; the in vitro (c) and in vivo (d, e) experiments of MPC [22] (color online).

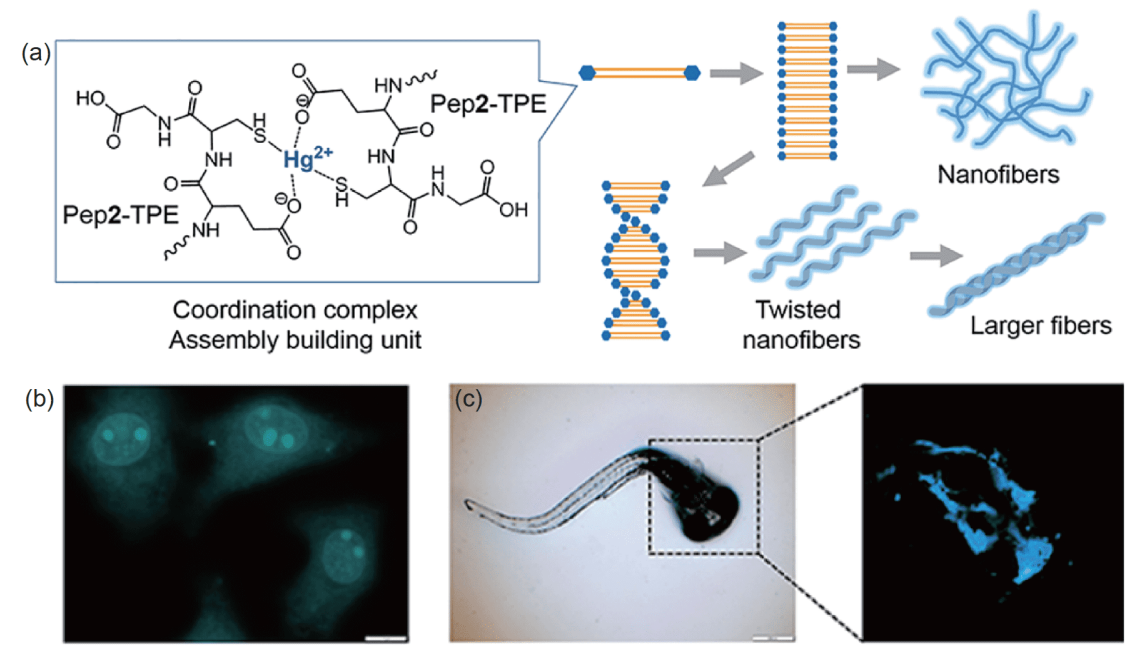

图 2 (a) 连接了PTE的 $\gamma$-谷胱甘肽分子与 $\mathrm{Hg}^{2+}$ 形成配位作用示意图; (b, c) 利用多肽组装体在 $\mathrm{HeLa}$ 细胞和斑马鱼中对 $\mathrm{Hg}^{2+}$ 检 测的活体实验结果 ${ }^{[24]}$ (网络版彩图)

Figure 2 (a) Diagram of coordination between $\mathrm{Hg}^{2+}$ and $\gamma$-glutathione linked to PTE; (b, c) the detection of $\mathrm{Hg}^{2+}$ in HeLa cell and Zebra fish of in vivo assembly materials [24] (color online).

\section{3 体内多肽自组装体在组织工程方面的应用}

体内多肽自组装体还可以应用于组织修复的研 究. 利用体内多肽自组装策略可以实现对常见疾病 心肌梗塞或椎间盘磨损的微创乃至无创修复 ${ }^{[25]}$.

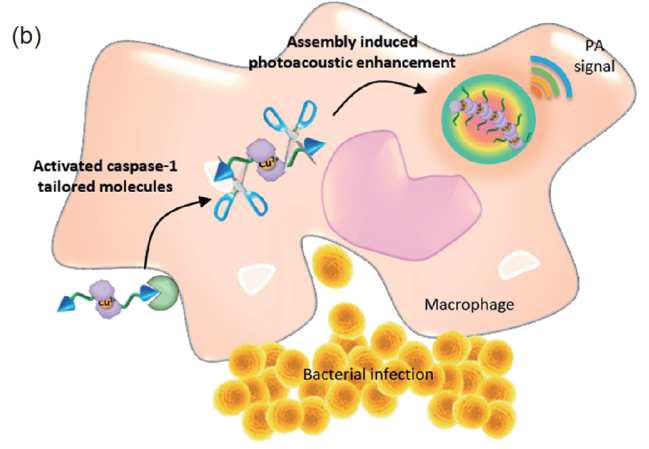

(e)

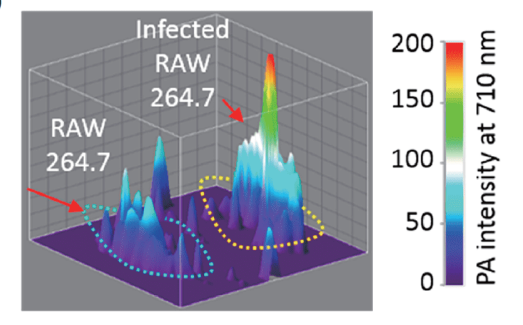


不是一种具有临床转化前景的研究手段. Christman课 题组 ${ }^{[25]}$ 则另辟蹊径，利用正常心脏部位脉管所具有的 高渗透和滞留效应(enhanced permeability and retention effect，EPR)，设计了一段含有对基质金属蛋白酶(matrix metalloproteinases, MMPs)具有特异性响应的多肽 (PLGLAG)的两亲性分子(图3(a))。该分子通过透析得 到的材料呈纳米囊泡状，经过血液循环和EPR效应在 发生心肌梗塞部位富集时，能被该部位过表达的 MMP-2和MMP-9酶特异性水解，原位形成纳米纤维的 组装结构(图3(b)), 从而将生物修复材料在心肌部位的 滞留时间从 $1 \mathrm{~h}$ 提高到 $7 \mathrm{~d}$ (图3(c)). 而通过心肌梗塞的 老鼠模型实验，也证明了该策略对心肌损伤修复的高 效性(图3(d)).

在组织修复方面，通过对体内多肽自组装体的设 计，还可以起到药物原位缓释作用. Wilcox课题组 ${ }^{[27]}$ 利用体内多肽自组装策略, 实现了对由于软骨素(葡萄 糖胺聚糖)流失而引起的椎间盘磨损治疗的微创化. 利 用多肽分子原位快速响应形成组装体的原理，可以显 著提高软骨素在目标部位的滞留时间，进而提高软骨 素对椎间盘磨损的修复作用. Wilcox课题组所采取的

(a)

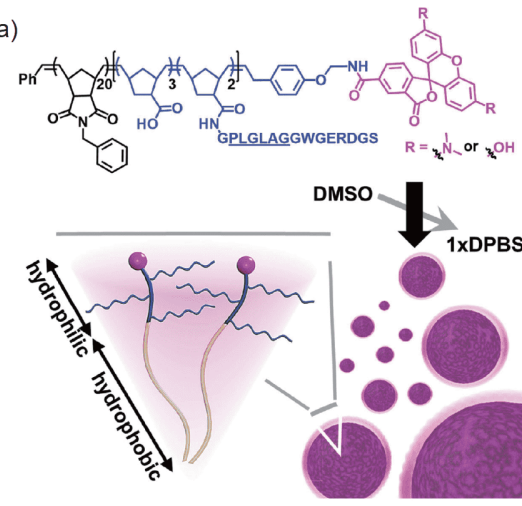

$1 \mathrm{~min}$

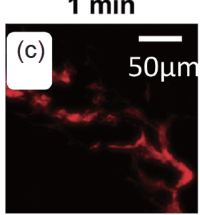

$1 \mathrm{hr}$
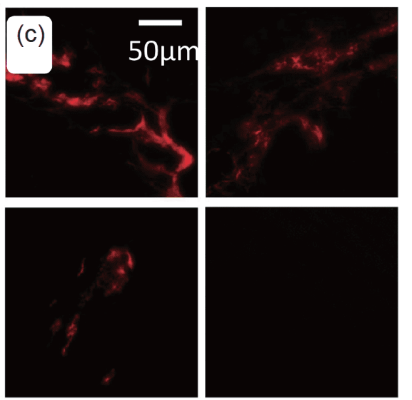

方法是先向病灶部位注射软骨素，而后再注入具有原 位响应形成水凝胶性质的多肽分子. 多肽分子与软骨 素在病灶部位原位共组装形成水凝胶，整个过程仅需 要利用针头注射, 实现了对椎间盘磨损的微创治疗.

利用多肽作为药物载体具有多种优势，因此该方 向得到广泛研究 ${ }^{[28-31]}$. 针对药物循环易流失、传统药 物载体药物负载释放过程过于复杂且效率低的弊端, 利用体内多肽自组装策略在病灶部位构筑药物原位释 放模型具有很好的应用优势. Zhong课题组 ${ }^{[32]}$ 设计了 一段对 $\mathrm{Zn}^{2+}$ 具有响应性的分叉型多肽 $\mathrm{E}_{3}-\mathrm{F}_{n}$, 用于治疗 前列腺癌症的药物输送平台. 具有 $\mathrm{Zn}^{2+}$ 响应组装性质 的多肽 $E_{3}-F_{3}$ 与亚德里亚霉素混合物溶液呈澄清状, 将 溶液注射进 $\mathrm{Zn}^{2+}$ 浓度远高于其他部位的前列腺部位 后，多肽分子与 $\mathrm{Zn}^{2+}$ 配位原位组装形成水凝胶同时将 药物分子锁定, 进而起到药物的精准缓释作用.

\section{4 体内多肽自组装体用于神经退行性疾病 的治疗}

随着我国老龄化的加剧，神经退行性疾病患病率
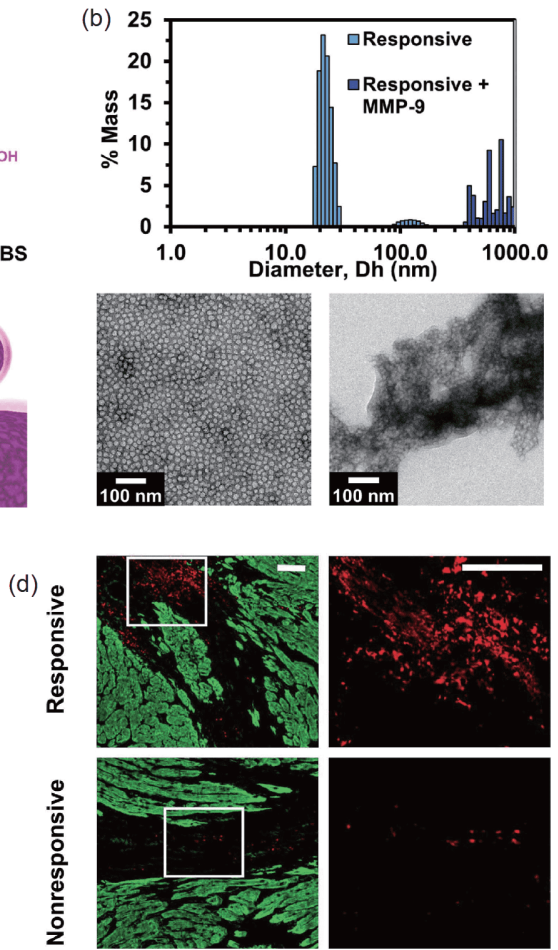

图 3 (a) 两亲性多肽高分子结构示意图; (b d) 体外与活体实验结果 ${ }^{[25]}$ (网络版彩图)

Figure 3 (a) Diagram of the peptide-polymer amphiphile; (b-d) results of in vivo and in vitro experiments [25] (color online). 
不断增长. 神经退行性疾病是一类大脑和脊髓神经元 细胞丧失的疾病状态，神经元细胞一般不会再生，过 度的损害可能是毁灭性的, 不可逆转的. 科学家们一 直在努力寻找神经退行性疾病的治疗方法. 本课题 组 ${ }^{[33]}$ 利用多肽纳米颗粒作为“纳米清道夫”来治疗阿尔 兹海默病的研究则为这种利用外源性多肽与体内多肽 进行共组装的研究指明了未来的研究方向. 阿尔兹海 默病主要是由淀粉样纤维 $\mathrm{A} \beta$ 为基础的细胞外自组装 纤维沉积和含有高磷的胞内神经原纤维的缠结所引起 的 ${ }^{[34]}$. 人体大脑在正常生理条件下也会产生 $A \beta$, 但是 自身免疫系统会将其进行清除，当清除的速率低于产 生的速率时, 就产生了纤维的堆积 ${ }^{[34]}$. 如何能够实现 对形成的沉积蛋白进行清除是治疗该类疾病的一大突
破口。

在研究中, 我们合成了一个多功能的纳米颗粒, 带 正电的壳聚糖的高分子为内核, 连接GKLVFF多肽片 段用于捕捉 $\mathrm{A} \beta$ 蛋白, 并修饰以自噬相关蛋白(Beclin-1) 多肽片段, 能够激活自噬(图4(a)). 在该结构中, PEG能 显著提高分子在水中的分散性，也提升该“清道夫”的 生物相容性和稳定性. 分子中的KLVFF能通过氢键作 用与A $\beta-42$ 进行共组装，而Beclin-1则能够激活自噬分 解共组装多肽组装体. 纳米清道夫通过氢键将 $A \beta$ 捕 获，复合体通过内吞作用进入细胞，清道夫上的受体 Beclin-1激活细胞自噬使得清道夫与 $\mathrm{A} \beta$ 蛋白一同被降 解(图4(b)). 体外实验以及小鼠的活体实验均证明了材 料对 $A \beta$ 蛋白高效的清理能力. 利用外源性引入的多肽

(a)

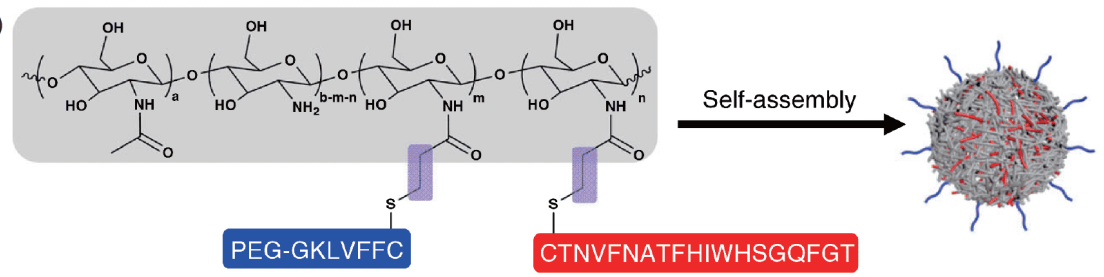

Molecular structure of nanosweeper

(b)

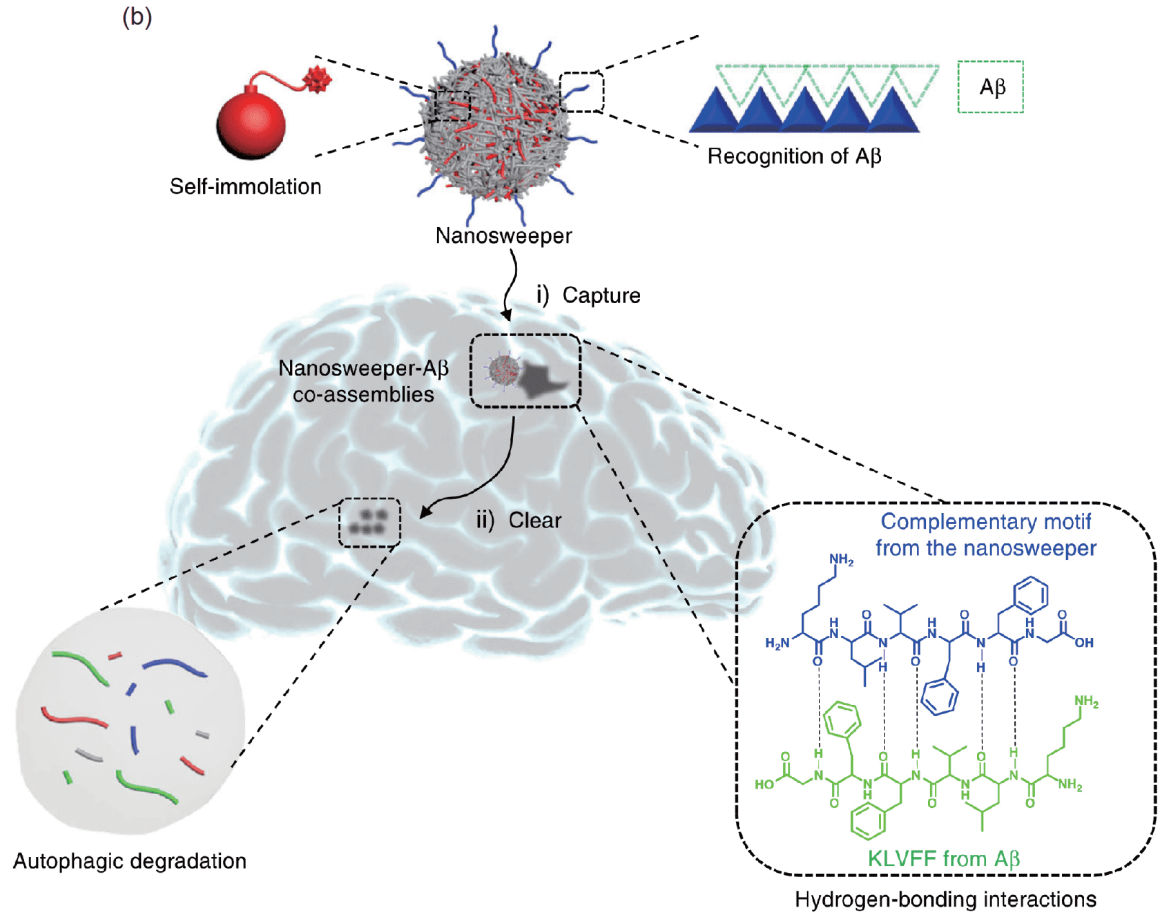

图 4 纳米清道夫的结构示意图(a)和纳米清道夫的工作机理(b) ${ }^{[33]}$ (网络版彩图)

Figure 4 Diagram of the structure of nano-sweeper (a), and the mechanism of the clearance of A $\beta$ by nano-sweeper (b) [33] (color online). 
与 $\mathrm{A} \beta-42$ 间通过氢键作用进行共组装形成纳米颗粒, 清除脑部产生的淀粉样纤维 $\mathrm{A} \beta$ 蛋白则为临床上该疾 病的治疗带来了希望.

\section{5 总结与展望}

合理设计体内原位自组装体结构以实现特定功 能, 在生物医学领域具有极大的潜在应用价值. 我们通 过活体自组装策略构筑的多肽自组装体在肿瘤以及非 肿瘤相关的重大疾病诊断和治疗方面发挥着重要作 用. 当然, 在体内多肽自组装体的研究中, 我们还面临 着一系列的困难. 首先, 活体内原位表征多肽组装体的 结构一直是一个难点. 虽然我们能通过体外实验很好 地模拟多肽及其剪切残基在体外的组装过程，但是活 体内的生理环境远比体外模拟的环境复杂. 从多肽分 子离开针头那一刻起的所有多肽分子在体内的物理化 学行为都脱离了我们的控制. 因此, 目前亟待解决的一
大难点是多肽分子在生物体内的靶向、响应、组装和 代谢的动力学行为控制和表征的研究.

此外，已有的天然多肽与通过蛋白修饰或改性获 得的多肽难以满足日益扩大的临床需要, 通过合成生 物学及合成化学手段发展更高效的合成、篎选手段 以得到具有更好组装性能、更精准特异性靶点和更 高稳定性的多肽是这个研究领域发展中必然经历的 阶段.

最后, 在发展新的功能性多肽的同时, 对已知多肽 结构的修饰改性以提高其生物利用度和改善其在体内 组装行为仍不失为一种改善多肽材料性能的有效手 段. 在这个过程中, 利用天然氨基酸在多肽或蛋白上进 行突变是一种能将对天然多肽影响降到最低的方式. 或者通过引入非天然氨基酸如 $\mathrm{D}$ 型氨基酸或 $\beta$ 及 $\gamma$ 氨基 酸等结构将有可能成为改善多肽自组装纳米材料生物 效应的另一突破口. 实现这一目标的前提是计算科学 和合成化学的进一步发展.

\section{参考文献}

1 Gao Y, Yang Z, Kuang Y, Ma ML, Li J, Zhao F, Xu B. Biopolymers, 2010, 94: 19-31

2 Gao X, Matsui H. Adv Mater, 2005, 17: 2037-2050

3 Pattabiraman VR, Bode JW. Nature, 2011, 480: 471-479

4 Wang L, Li LL, Ma HL, Wang H. Chin Chem Lett, 2013, 24: 351-358

5 Chen Y, Liu L. Adv Drug Deliver Rev, 2012, 64: 640-665

6 Liu Y, Du J, Yan M, Lau MY, Hu J, Han H, Yang OO, Liang S, Wei W, Wang H, Li J, Zhu X, Shi L, Chen W, Ji C, Lu Y. Nat Nanotech, 2013, 8: 187-192

7 Ma Y, Huang J, Song S, Chen H, Zhang Z. Small, 2016, 12: 4936-4954

8 Rao L, Bu LL, Cai B, Xu JH, Li A, Zhang WF, Sun ZJ, Guo SS, Liu W, Wang TH, Zhao XZ. Adv Mater, 2016, 28: 3460-3466

9 Wang Z, An HW, Hou D, Wang M, Zeng X, Zheng R, Wang L, Wang K, Wang H, Xu W. Adv Mater, 2019, 31: 1807175

10 Chou LYT, Ming K, Chan WCW. Chem Soc Rev, 2011, 40: 233-245

11 Shang Y, Zhi D, Feng G, Wang Z, Mao D, Guo S, Liu R, Liu L, Zhang S, Sun S, Wang K, Kong D, Gao J, Yang Z. Nano Lett, 2019, 19: 15601569

12 Du W, Hu X, Wei W, Liang G. Bioconjugate Chem, 2018, 29: 826-837

13 Acar H, Srivastava S, Chung EJ, Schnorenberg MR, Barrett JC, LaBelle JL, Tirrell M. Adv Drug Deliver Rev, 2017, 110-111: 65-79

14 Zhang D, Qi GB, Zhao YX, Qiao SL, Yang C, Wang H. Adv Mater, 2015, 27: 6125-6130

15 Lin YX, Wang Y, Wang H. Small, 2017, 13: 1700996

16 Qi GB, Gao YJ, Wang L, Wang H. Adv Mater, 2018, 30: 1703444

17 Li W, Wang D, Shi X, Li J, Ma Y, Wang Y, Li T, Zhang J, Zhao R, Yu Z, Yin F, Li Z. Mater Horiz, 2018, 5: 745-752

18 Li LL, Qiao ZY, Wang L, Wang H. Adv Mater, 2018, 440: 1804971

19 Ulijn RV, Smith AM. Chem Soc Rev, 2008, 37: 664-675

20 Hai Z, Wu J, Saimi D, Ni Y, Zhou R, Liang G. Anal Chem, 2018, 90: 1520-1524

21 Li LL, Ma HL, Qi GB, Zhang D, Yu F, Hu Z, Wang H. Adv Mater, 2016, 28: 254-262 

ONE, 2018, 13: e0204164

\section{7-5552}

H, Gu Z, An H, Chen C, Chen J, Cui R, Chen S, Chen W, Chen X, Chen X, Chen Z, Ding B, Dong Q, Fan Q, Fu T, Hou D, Jiang Q, Ke H, Jiang X, Liu G, Li S, Li T, Liu Z, Nie G, Ovais M, Pang D, Qiu N, Shen Y, Tian H, Wang C, Wang H, Wang Z, Xu H, Xu JF, Yang X, Zhu S, Zheng X, Zhang X, Zhao Y, Tan W, Zhang X, Zhao Y. Sci China Chem, 2018, 61: 1503-1552

32 Tao M, Xu K, He S, Li H, Zhang L, Luo X, Zhong W. Chem Commun, 2018, 54: 4673-4676

33 Luo Q, Lin YX, Yang PP, Wang Y, Qi GB, Qiao ZY, Li BN, Zhang K, Zhang JP, Wang L, Wang H. Nat Commun, 2018, 9: 1802

\title{
Progress of in vivo self-assembled peptide nanomaterials for theranostics of non-cancerous diseases
}

\author{
Xiang-Zhong Zeng ${ }^{1,2}$, Hong-Wei An ${ }^{2,3}$, Hao Wang ${ }^{3 *}$ \\ ${ }^{1}$ Academy of Advanced Interdisciplinary Studies, Peking University, Beijing 100871, China \\ ${ }^{2}$ CAS Key Laboratory for Biomedical Effects of Nanomaterials and Nanosafety, CAS Center for Excellence in Nanoscience, National Center for \\ Nanoscience and Technology of China, Beijing 100190, China; \\ ${ }^{3}$ Institute of High Energy Physics, Chinese Academy of Sciences, Beijing 100049, China \\ *Corresponding author (email: wanghao@nanoctr.cn)
}

\begin{abstract}
In vivo peptide self-assembled nanoprobes and nanomedicines based on the "in vivo self-assembly" strategy are widely used in the diagnosis and therapy of tumors due to their high specificity and bioavailability. Based on the advantages of peptide self-assembling nanomaterials in vivo, they have also been used in other biomedical fields in addition to tumor diagnosis and therapy, such as accurate detection of bacterial infections, tissue repair, and therapy of neurodegenerative disease. This article briefly reviews the application of peptide self-assembled nanomaterials in vivo in other major diseases except the diagnosis and therapy of tumors.
\end{abstract}

Keywords: in vivo self-assembly, nanomaterials, peptide, non-cancerous diseases

doi: $10.1360 /$ SSC-2019-0021 